This is the post peer-review accepted manuscript of:

C. Silvano et al., "Supporting the Scale-Up of High Performance Application to Pre-Exascale Systems: The ANTAREX Approach," 2019 27th Euromicro International Conference on Parallel, Distributed and Network-Based Processing (PDP), Pavia, Italy, 2019, pp. 116-123. doi: 10.1109/EMPDP.2019.8671584

The published version is available online at: https://ieeexplore.ieee.org/abstract/document/8671584

(C) 2019 IEEE. Personal use of this material is permitted. Permission from IEEE must be obtained for all other uses, in any current or future media, including reprinting/republishing this material for advertising or promotional purposes, creating new collective works, for resale or redistribution to servers or lists, or reuse of any copyrighted component of this work in other works 


\title{
Supporting the Scale-up of High Performance Application to Pre-Exascale Systems: The ANTAREX Approach
}

\author{
Cristina Silvano ${ }^{\bigotimes}$, Giovanni Agosta ${ }^{\bigotimes}$, Andrea Bartolini $^{\star}$, Andrea R. Beccari $^{\ddagger}$, Luca Benini ${ }^{\dagger}$, \\ Loïc Besnard ${ }^{\ddagger \ddagger}$, João Bispo ${ }^{\S}$, Radim $\mathrm{Cmar}^{\dagger+}$, João M. P. Cardoso ${ }^{\S}$, Carlo Cavazzoni ${ }^{\natural}$, Daniele Cesarini ${ }^{x}$,

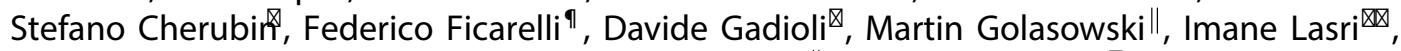 \\ Antonio Libri ${ }^{\dagger}$, Candida Manelf ${ }^{\ddagger}$, Jan Martinovičll, Gianluca Palermo ${ }^{\rrbracket}$, Pedro Pinto ${ }^{\S}$, \\ Erven Rohou ${ }^{\mathbb{}}$, Nico Sanna , Kateřina Slaninovál, Emanuele Vitali ${ }^{\mathbb{}}$ \\ ${ }^{{ }_{D}}{ }_{\text {DEIB }}$ - Politecnico di Milano, ${ }^{\dagger} \| I S$ - Eidgenössische Technische Hochschule Zürich, ${ }^{\ddagger}$ Dompé Farmaceutici SpA, \\ ${ }^{\S}$ FEUP - Universidade do Porto, "CINECA, \|IT4Innovations, VSB - Technical University of Ostrava,

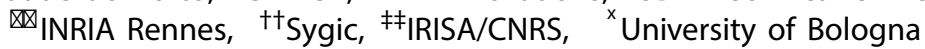

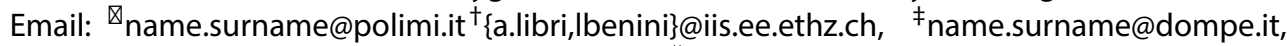

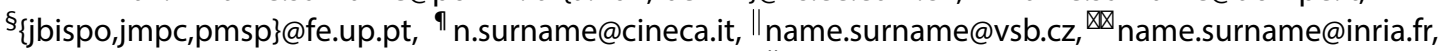

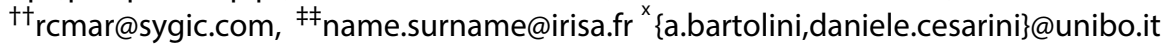

\begin{abstract}
The ANTAREX project developed an approach to the performance tuning of High Performance applications based on an Aspect-oriented Domain Specific Language (DSL), with the goal to simplify the enforcement of extra-functional properties in large scale applications. The project aims at demonstrating its tools and techniques on two relevant use cases, one in the domain of computational drug discovery, the other in the domain of online vehicle navigation. In this paper, we present an overview of the project and ofits main achievements, as well as of the large scale experiments that have been planned to validate the approach.

Keywords-High Performance Computing, Autotuning, Adaptivity, DSL, Compilers, Energy Efficiency
\end{abstract}

\section{I NTRODUCTION}

The design and optimisation of applications for High Performance Computing systems is extremely challenging, especially when taking into account the need for energy efficiency and extreme scalability. Indeed, to achieve an Exascale supercomputer, a radical improvement in energy efficiency is necessary, as the power demand under current technology constraints would reach hundreds of $\mathrm{MW}$, against a state target of 20-30 MW. According to the Green500 list ${ }^{1}$, which ranks supercomputers on the basis of the GigaFlops per Watt figure of metric, the "most green" supercomputer SHOUBU SystemB installed in Japan exceeds 18 GigaFlops/W during its 857TeraFlop/s Linpack performance run. The top positions in Green500 are all occupied by heterogeneous systems based on high-performance processors and co-processors such as the latest NVIDIA Volta GV100 GPU and PEZY SC2 accelerator to further accelerate the computation. The dominance of heterogeneous systems in the Green500 list is expected to continue for the next coming years to reach the target of $20 \mathrm{MW}$ Exascale supercomputers. Furthermore, even the

${ }^{1}$ www.green500.org June 2018
Top500 list ${ }^{2}$, which measures pure performance, is dominated by the Summit supercomputer installed in the USA. This reaches a peak performance of over 187 PetaFlops with a power envelop ofless than $9 \mathrm{MW}$ - still above the target for Exascale.

To fullfil the Exascale target, a goal that the European Union aims at reaching by $2023^{3}$, energy-efficient supercomputers need to be coupled with a radically new software stack. This stack should be capable of exploiting the benefits offered by architecture heterogeneity at different abstraction levels to meet the scalability and energy efficiency required by the Exascale era. Furthermore, the current development model where the HPC center staff directly supports the development of applications will become unsustainable in the long term, due to the inherent challenges of developing applications for heterogeneous systems. Thus, the availability of effective standard programming languages and APIs is crucial to provide migration paths towards novel heterogeneous HPC platforms as well as to guarantee that developers work effectively on these platforms.

To conclude, heterogeneous systems currently dominate the top of the Top500 and Green500 lists and this dominance is expected to be a trend for the next coming years to reach the target of 20MW Exascale supercomputers. To fullfil the 20MW target, energy-efficient heterogeneous supercomputers need to be coupled with radically new software stacks to exploit the benefits offered by heterogeneity at all levels (supercomputer, job, node) and to meet the scalability and energy efficiency required by the Exascale era.

Goals of the project. The ANTAREX [ 1, 2, 3, 4] project

\footnotetext{
${ }^{2}$ www.top500.org June 2018

${ }^{3}$ https://www.consilium.europa.eu/en/press/pressreleases/2018/06/25/supercomputers-council-agrees-to-develop-high-techinfrastructure/
} 
aims at providing a holistic approach spanning all the decision layers composing the supercomputer software stack and exploiting effectively the full system capabilities, including heterogeneity and energy management. The main goal of ANTAREX is to express the application self-adaptivity and to runtime manage and autotune applications for green heterogeneous HPC systems up to the Exascale level by means of a DSL. The use of a DSL allows the introduction of a separation of concerns, where self-adaptivity and energy efficient strategies are specified separately from the application functionalities. This is promoted by the definition of a DSL inspired by aspect-oriented programming concepts for heterogeneous systems. The DSL is based on previous efforts regarding the LARA language $[5,6]$ and it makes possible to express at compile time the adaptivity/energy/performance strategies and to enforce at runtime application autotuning as well as resource and power management. The goal is to support parallelism, scalability and adaptivity in a dynamic workload by exploiting the full system capabilities (including energy management) for emerging large-scale and extremescale systems, while reducing the Total Cost of Ownership (TCO) for companies and public organizations.

Key innovations. The ANTAREX approach provides: (1) A new DSL for expressing adaptivity and autotuning strategies; (2) Enabling the performance/energy control capabilities by tuning software knobs (including application parameters, code transformations and code variants); (3) Designing scalable and hierarchical optimal control-loops capable of dynamically leveraging them together with performance/energy control knobs at different time scales (compile-, deploy- and run-time) to always operate the supercomputer and each application at the maximum energy-efficient and thermally-safe point. This can be done by monitoring the evolution of the supercomputer as well as the application status and requirements and bringing this information to the ANTAREX energy/performance-aware software stack.

The project is driven by two use cases taken from highly relevant HPC application scenarios: (1) a biopharmaceutical application for drug discovery deployed on the Marconi system at CINECA and (2) a self-adaptive navigation system for smart cities deployed server-side on the Anselm and Salomon systems provided by IT4Innovations National Supercomputing Center.

The ANTAREX Consortium. To achieve the ANTAREX goals, a broad range of technical expertise is needed. As a consequence, the ANTAREX Consortium comprises some of the foremost institution in European research. The consortium is led by Politecnico di Milano, one of the largest technical universities in Europe, and comprises top-ranked academic partners such as ETH Zürich (with additional support from University of Bologna), Universidade do Porto, and INRIA (with additional support from IRISA/CNRS). Real-world applications are provided by one of the leading biopharmaceutical companies, Dompè, and by the top navigation software company in Europe, Sygic. To effectively validate the tool flow, as well as to support the deployment and scale-up

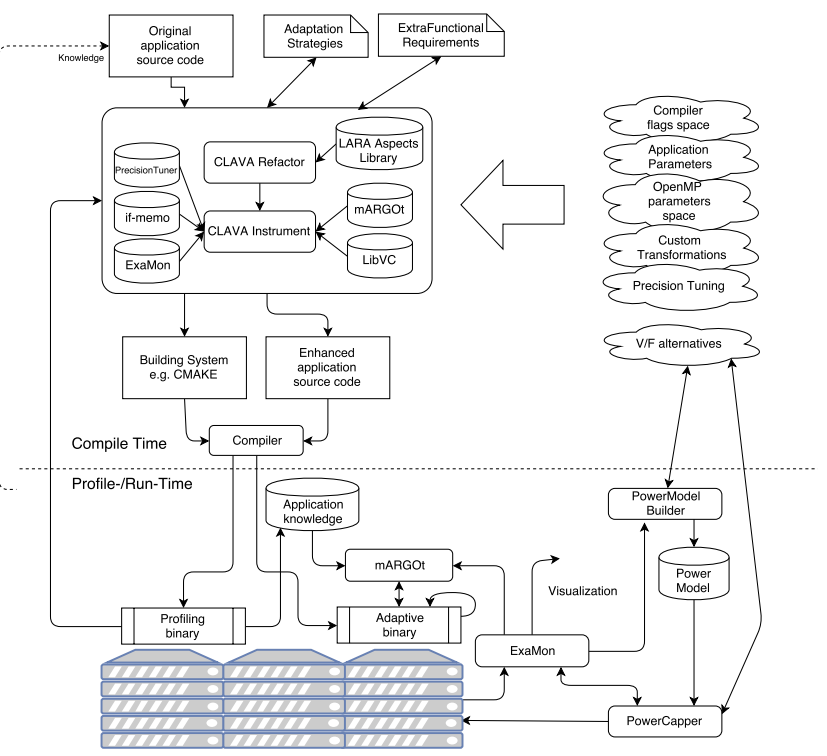

Fig. 1. The ANTAREX Tool Flow

of the target applications, the consortium also includes two supercomputing centers, CINECA in Italy and IT4Innovations in Czech Republic. CINECA's Marconi supercomputer is ranked 18 in the Top500 as of June 2018, making it the 3rd most powerful supercomputer in the European Union, while IT4Innovations' Salomon is ranked 139.

Organization of the paper The rest of this paper is organized as follows. In sectionll we outline the ANTAREX approach. In sectionsIII and IV we introduce the target platforms and use case scenarios employed for the validation of the ANTAREX tools and methodologies. Finally, in section V we draw some conclusions.

\section{T HE ANTAREX A PPROACH}

Figure 1 shows the ANTAREX approach through its tool flow, covering both the design-time and the runtime operation. The functionality of the target application is expressed through a standard programming language - the current tools support both $\mathrm{C}$ and $\mathrm{C}++-$ and can therefore include legacy code. On the other hand, the extra-functional aspects of the application are expressed through a separate specification, written in ANTAREX DSL [ 4]. ANTAREX DSL is based on LARA [ 7], and then extended to provide support for parallelisation, mapping, precision tuning and adaptivity strategies. In this way, the expression of extra-functional concerns is fully decoupled from the functional code, allowing the application domain expert and the performance tuning expert to operate as independently as possible.

The DSL specification is weavedinto the functional code at compile time, through a source-to-source transformation tool, Clava. This tool performs a refactoring of the application code based on the aspects provided, and instruments it with the necessary calls to other components of the tool flow. 
a) Dynamic Compilation: Partial dynamic which the runtime controller selects the actual configuration (re)compilation is a technique used as part of the continuous depending on the workload, as well as the system parameters. program optimisation \$]. It allows the compiler to further optimise the code during the execution oflong runs of an application, which are typical of HPC scenarios. While most high-level languages include mechanisms for selective compilation which can be exploited for fine tuning the dynamic compilation options, e.g. for hiding compilation latencies [9], the C/C++ applications commonly used in HPC scenarios generally lack this option. Some support is provided by domain-specific tools, such as RuntimeCompiledC++1[0], which focuses on interactive modification and recompilation of program fragments by the programmer. To enable partial dynamic compilation, ANTAREX DSL aspects can introduce calls to a support library, libVC [11], thus weakening the boundary between compile-time and runtime, and enabling continuous optimisation.

b) Precision Tuning: Among the optimisation techniques, ANTAREX developed tools for precision tuning, which has emerged as a promising approach to improve power/performance trade-offs. Precision tuning originates from the fact that many applications can tolerate some loss of quality during computation, as in the case of media processing (audio, video and image), data mining, machine learning, etc. Error-tolerating applications are increasingly common in the emerging field of real-time HPC. In ANTAREX, we explored both precision tuning of floating point computation on GPGPU accelerators [12] and floating to fixed point conversion, followed by tuning of the fixed point representation in terms of bit width and point position13, 14].

c) Memoization: For a long time, memoization has been employed as a performance optimization technique, albeit primarilyin functional languages. A survey of techniques to handle memoization at programming language level, as well as references to applications can be found in 1 5]. More recently, memoization has been shown as a promising path for energy saving in computation-intensive workload $\$ \Phi, 17]$. In ANTAREX, a library to support memoization $[18,19]$ has been integrated in the DSL, allowing automated exploration of memoization opportunities.

d) System Monitoring:System monitoring is a key aspect of HPC infrastructures. In ANTAREX, we leverage the ability of processing elements to provide performance metrics at hardware level through thexaMon tool [20]. This provides a virtualisation of the performance and power monitoring, decoupling sensor readings from sensor value use. This approach increases the scalability of the monitoring, and at the same time provides an easy access to monitoring APIs through the ANTAREX DSL.

e) Performance Tuning and Power Management:Techniques employed to control the performance of one or more applications according to system parameters such as power consumption have been developed in the last decade in both high performance and embedded computing fieldsi,[22]. Typically, a design-time phase leverage optimisation toola $B]$ to allow the identification of specific operating points, among

In ANTAREX, at runtime, the mARGOt tool [24][25] configures the available software knobs (application parameters, code transformations and code variants) according to the runtime information coming from application self-monitoring and system monitoring, thus creating an autotuning control loop. Finally, the runtime power managerPowerCapper, is used to control the resource usage for the underlying computing infrastructure in evolving conditions 26, 27].

At runtime, the application control code, thanks to the design-time phase, now contains also runtime monitoring and adaptivity strategy code derived from the DSL extra-functional specification. Thus, the application is continuously monitored to guarantee the required Service Level Agreement (SLA), while the communication with the runtime resource-manager takes place to control the amount of processing resources needed by the application. The application monitoring and autotuning is supported by a runtime layer implementing an application level collect-analyse-decide-act loop.

\section{T ARgET PLATFORMS}

The target platforms are two PetaFlop class systems, the CINECA's top-level supercomputer, Marconi, and IT4Innovations Salomon supercomputer.

\section{A. CINECA Platform \& Roadmap}

CINECA, to further its institutional mission and to support the competitiveness of the Italian research infrastructure, including its participation to the international supercomputing enviroment in Europe (PRACE), operates an evolving, massive infrastructure aiming at a convergence of computing and data handling tasks. CINECA aims at growing its infrastructure to bridge the gap to Exascale around 2023, as well as to support emerging paradigms such as Big Data analytics, realtime HPC, and cloud HPC.

The current supercomputing machine (Marconi), in production from the second-half of 2016, is set up as a scalable hybrid cluster starting at 10 PFLOPS, and currently benchmarked at a peak of 16 PFPLOS $^{4}$. The CINECA roadmap will lead to a scale up of the Marconi machine to over 20 PFLOPS in 2019, and up to over 200 PFLOPS by 2022, with a power envelop growing from the current $3 \mathrm{MW}$ to around $10 \mathrm{MW}$.

However, during the last ten years, the path following to high-end computing and data managing has revealed a major constraint in the exponential growth of power consumption. Thus, it is now commonly accepted that a limit of $20 \mathrm{MW}$ will need to be imposed for the first exaFLOPS supercomputing system. With this limit in mind, CINECA is modelling its strategy for future HPC/DA systems in order to experiment, design and deploy energy-aware supercomputing facilities. To this end, CINECA has been involved in several projects at national and EU level to take advantage of the most promising techniques for limiting the overall requirements of energy-tosolution computing workflow $48,29,30,31]$.

${ }^{4}$ https://www.top500.org/list/2018/06 
In the context of ANTAREX, CINECA is employing the A2 partition of Marconi ${ }^{5}$, which includes 3,600 nodes, each one equipped with a 68-cores Intel Xeon Phi7250 (Knights Landing), clocked at $1.4 \mathrm{GHz}$, for a total of 244,800 cores. Each node is equipped with $16 \mathrm{~GB} /$ node MCDRAM +96 $\mathrm{GB} /$ node DDR4. The partition has a peak performance of 11 PFLOPS.

\section{B. IT4Innovations Platform \& Roadmap}

IT4Innovations as the National Supercomputing Center of the Czech Republic operates two HPC clusters as of 2018. The first one is a 2 PFLOP/s system called Salomon having 1008 nodes based on Intel Haswell CPUs ${ }^{6}$. Half ofits computing power is provided by Intel Xeon Phi accelerator' based on the Knights Corner architecture. It uses InfiniBand FDR56 interconnect in 7D Enhanced hypercube topology.

The second cluster is a smaller $94 \mathrm{TFLOP} / \mathrm{s}$ system called Anselm It has 209 nodes based on Intel Sandy Bridge CPUs ${ }^{8}$. It also contains $23 \mathrm{GPU}$ accelerated node's and 4 MIC accelerated nodes.

Each cluster has its own dedicated storage available, including distributed filesystem LUSTRE. In addition to this, each cluster has a set of nodes for virtualization with its own storage which can be used to run virtual machines providing auxiliary services. The ANTAREX project used both machines, including their virtualization infrastructure, extensively.

During the project a small portion (18 nodes) from the Anselm cluster has been removed from the PBS scheduler queues and dedicated entirely to the development and integration activities related to the UC2. The dedicated partition has been isolated in a private network segment accessible only via a VPN operated by IT4Innovations. Access to the infrastructure has been restricted only for members of the ANTAREX consortium involved in the UC2 activities.

The main purpose of the dedicated partition was to gain a privileged access to the computing hardware for testing of various network settings and running performance monitoring tools which require privilege escalation. It has been used mainly to verify and validate the server-side routing service using the traffic simulator. The integration of the mARGOt autotuner has been also tested there. The next step is to use the dedicated partition to test tools which require privilege escalation, mainly mARGOt autotuner with energy constraints measured by PAPI and other related experiments.

The remaining production part of the Anselm cluster has been used to test the scalability and performance of the experimental server-side routing service. It is also used to verify and validate the developed service using the traffic simulator and large number Sygic navigation app instances.

\footnotetext{
${ }^{5}$ https://wiki.u-gov.it/confluence/pages/viewpage.action?pageld=131696536

${ }^{6} 2$ x Intel Xeon E5-2680v3, 2.5 GHz, 12 cores, 128 GB RAM per node

${ }^{7} 2 x$ Intel Xeon Phi 7120P, 61 cores, 16 GB RAM

$82 \times$ Intel Sandy Bridge E5, $2.4 \mathrm{GHz}, 8$ cores, 64/96 GB RAM, per node

${ }^{9} \mathrm{NVIDIA}$ Kepler K20m

${ }^{10}$ Intel Xeon Phi 5110P
}

The Salomon cluster is used mainly for computing whatif scenarios using the betweenness centrality algorithm. In addition to this, it has been used to test the performance and scalability of the algorithm's multi-node implementation as well as to verify and validate tools developed by INRIA and integrated in the algorithm.

\section{A PPLICATION SCENARIOS}

To demonstrate the impact of the ANTAREX toolchain, the project leverages two applications from emerging domains.

The first application is drawn from the computational chemistry domain, and aims at speeding up dramatically the early phases of the drug discovery process, allowing a fast screening of a large amount of potential drug molecules. The second application is drawn from the navigation system domain, and aims at introducing a server-side aspect to the navigation, with the final goal of providing directions to vehicles in a coordinated manner, to ensure a near-optimal transit time to each vehicle.

The two applications are well positioned, since they represent two critical societal challenges, and map to two different classes of HPC applications - the massively parallel class, and the real-time HPC class.

\section{A. Computer Accelerated Drug Discovery System}

The goal of a drug discovery process is to find novel drugs starting from a huge exploration space of possible molecules. Typically, this process involves several in vivo, in vitro and in silico tasks ranging from chemical design to toxicity analysis. One of these tasks is molecular docking. This consists in estimating the three-dimensional pose of a given molecule, namedligand, when it interacts with the target protein. Since the ligand is much smaller than the target protein, we focus a small region of the target protein (or receptor), nampocket (or binding site). Given the three-dimensional pose of the ligand within the pocket, we are able to estimate the strength of the chemical and physical interactions between the ligand nd the pocket by computing a geometric fitting score.

The evaluation of the pose of one individual ligand is independent from the evaluation of all the other candidates. Given that in drug discovery the number ofligands to be analyzed exceeds the billion of units, this issue can be considered embarrassingly parallel. However, to find the threedimensional pose that the ligand has when it interacts with the pocket, we have to deal with a large number of degrees of freedom.

As evaluating the chemical and physical interactions between the ligand and the pocket is a computationally intensive problem, we need to split the pose prediction task from the virtual screening task. The pose prediction task focuses on providing the best pose for a given ligand within a given binding site, whereas the virtual screening task aims at selecting among a huge database of candidates a small set of promising ligands which best fit the given binding site. A remarkable difference between the pose prediction and the virtual screening task lies in the different approach used to estimate the chemical and 
physical interactions between the ligand and the pocket on one hand, and the approach used to determine the pose to be evaluated on the other.

The estimation of the interactions between the ligand and the pocket can be done with either a geometrical or a pharmacophoric approach. The geometrical approach estimates the ligand-pocket interactions by only using the shape and volume information, whilst the pharmacophoric approach evaluates the actual chemical and physical interactions. Although the best solution according to a pharmacophoric approach has also a very good geometrical score, the best geometrical solution does not guarantee to be either a valid solution or a good solution from a pharmacophoric perspective. On the other side the geometrical evaluation is orders of magnitude faster than the pharmacophoric one.

At the same time, we have two alternative ways of estimating the poses to be evaluated: a pure geometrical and a molecular dynamic based approach. The former is based on playing on the molecule flexibility to geometrically fit the ligand in the pocket, while the latter is based on a molecular simulation that places the atoms on a minimal energy point. Also in this case, while the geometrical approach provides a faster time to solution, the other one is more accurate in finding better poses.

The use of molecular simulations together with a pharmacophoric estimation, which we can observe in the pose predic tion task, is the most accurate but also most computationallyintensive approach. It is regularly exploited on the pose prediction task. The combined geometrical approach on the other side can be exploited for the virtual screening phase.

In ANTAREX, we focused on the combination of the geometrical approaches with the goal of screening a huge amounts ofligand. Indeed, we developed within LIGEN a completely new module, namedGeoDock, for molecular docking that is based on geometric evaluations only. This reduces a lot the cost of the docking process by enabling the user to increase the size of the ligand database to be processed during the virtual-screening task. In particular, it is possible to process each ligand of the chemical library in an independent way, and given the size of the target ligand library, the problem can be considered embarrassing paralleGeoDock is a typical example of batch application optimized for homogeneous HPC platforms that can scale up to exascale.

a) Application of the ANTAREX Tools: We applied application autotuning approaches to the de-novo computer accelerated drug design based on LiGenDock. The goal was to demonstrate that it is possibile to optimize both energy-tosolution and time-to-solution by means of an improved task processing strategy and by exploiting energy-awareness. We exploited the low-intrusiveness and low-overhead features of EXAMON [ 20] to keep track continuously of the application behavior and ofits effect on the target architectures. This has been used to support manual and automatic tuning especially in the context of power optimization, and to deliver application components to available resources that best fit the computational complexity of each task.

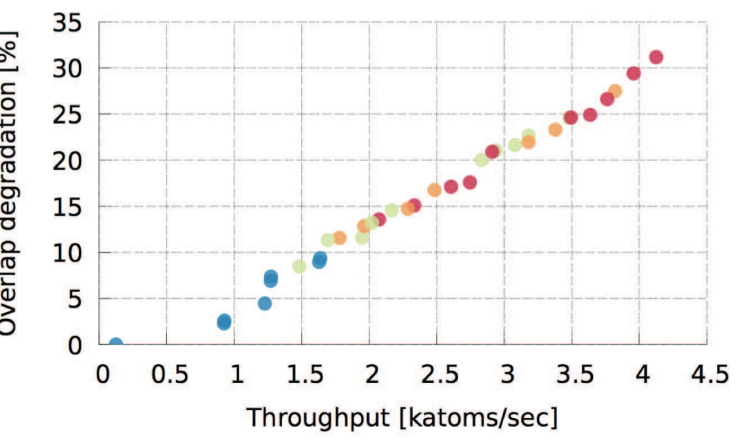

Fig. 2. Pareto solution of GeoDock while changing the application configuraitons

In the design phase, we used the precision tuning tools to understand the impact of the use of floating point arithmetics on the accuracy of the computation of the scoring function and for the screening of the most promising poses of the ligands. The analysis performed outlined that the original precision was not always necessary, as the accuracy needed to distinguish among good solutions (from a geometrical perspective) in most of the cases is much lower. Even lower precision was needed to classify the solutions only in terms qfromising/notpromising The parameterization of the application, and in particular the possibility to play with different levels of accuracy, has been used at deploy time to configure each run according to the target database to be screened, the amount of resources available for the experiment and the time-to-solution planned. Figure 2 shows the performance throughpult and accuracy (overlap degradation tradeoff curve obtained by exploring the tuning alternatives of the target application while running on a single node. This trade-off curve is used by mARGOt 25] for the autotuning phase.

b) Validation: Figure 3 and 4 show a preliminary analysis of the weak and strong scaling for theoDock module while running on the MARCONI-A2 partition composed of Intel KNL nodes. In particular, Figure 3 shows the elapsed time of a GeoDock run while considering 100 ligands. The considered number of nodes ranges from 8 to 128, while each node runs $68 \mathrm{MPI}$ processes. From the figure it is possible to notice how starting from 128 nodes we see a bad weak-scaling behavior. While the small oscillations up to 64 depend on the different types ofligands we are screening (the computation is data-dependent), at 128 nodes we noticed a bottleneck on the I/O that almost doubled the execution time. These initial results have been used to optimize the $\mathrm{l} / \mathrm{O}$ part of t6eoDock module. On the contrary, Figure 4 shows how the application throughput varies when the number ofligands per MPI process increase and when we consider a setup with 32 nodes and 68 $\mathrm{MPI}$ procesess per node. Given that there is no synchronization inside the application, this experiment can be considered as a proxy of a strong scaling. In particular we can notice a smooth performance degradation starting from around 30 ligands per 
MPI process. As for the previous case, the small oscillations we can observe for values larger than 30 are due to the data variability.

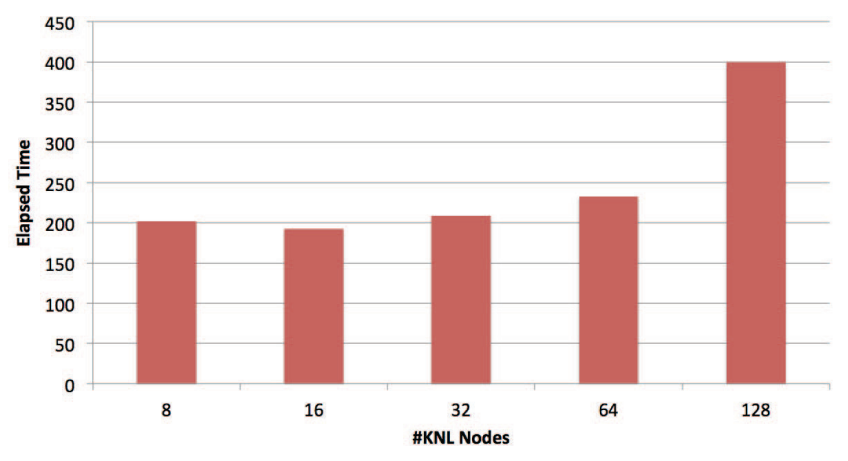

Fig. 3. Weak scaling analysis for the GeoDock module.

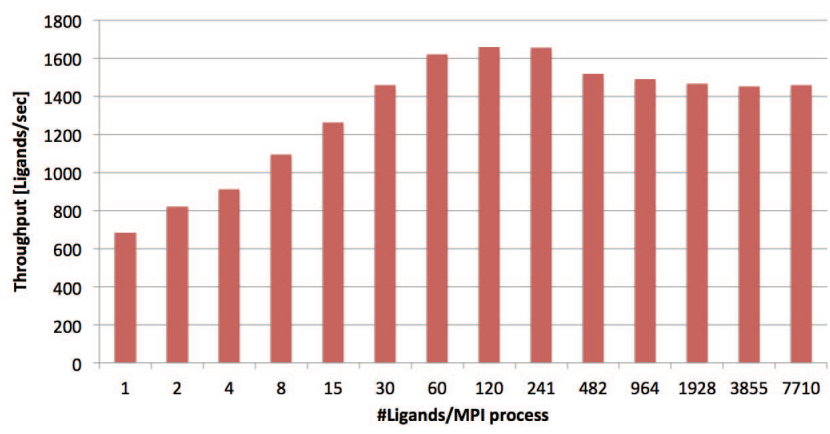

Fig. 4. Strong scaling analysis for theGeoDock module.

\section{B. Self-adaptive Navigation System in Smart Cities}

The main aim of this use case is to integrate and verify the ANTAREX tools in an experimental server-side navigation system. The navigation system provides an on-line navigation service which maintains a global view on the state of the road network in a given geographical area as well as on the means for managing hypothetical traffic scenarios which can occur in the road network.

Many modern cities need to cope with rapid growth of traffic volume in the recent years. Expanding the road network is a very expensive and long-term task and in some cases (city centers, dense agglomerations) virtually impossible due to lack of available space. The other option is to divert the traffic in a way which would be nearly optimal for each individual car. This can be achieved by various means such as dynamic road signs and large displays, lane switching or dynamic signaling plans for junctions. These means can be inefficient because it is impractical to deploy them on every street in a city. Another approach is to implement an on-line navigation service and use smart phones as endpoints for such system. Modern phones can indeed use such system. The GPS receiver is used to get actual position with sufficient precision while mobile data connection can be used to communicate with the service in real time. Both approaches can be combined as the serverside routing service can be enriched by data from various other sources (weather models, stationary traffic sensors) and the traffic infrastructure can take advantage from results of this data fusion to provide more precise information for the dynamic elements of the infrastructure (junction signaling, overhead highway displays and others).

The communication with the service can be bi-directional, as the mobile phone obtains the optimal route from the system and can provide feedback which would enhance the service awareness of the traffic network current state. To ensure the agreed quality of service for the user, the smartphone application should be able to switch between offline and online navigation, in case there is a lack of mobile phone coverage. It should also optimize the amount of communication needed for sending the feedback.

In the context of UC2, the server-side routing service is developed by IT4Innovations in collaboration with Sygic which provides its navigation smartphone app as a clientside endpoint for the routing service. Part of the server-side routing service is also a web-based interface for computing a betweenness centrality of a road network which can then be used to model behavior of the traffic under certain conditions [32]. The web-based interface allows the defition of a number of what-if scenarios, including road closure, an event which affects speed on roads in a given area and other options. The system is seamlessly integrated with HEAppE service which is used to submit the computation job with the scenarios on the cluster B3]. Results of the computation can be accessed using the same interface when the job is finished.

a) Application of the ANTAREX Tools: UC2 makes use mainly of the following tools, LARA DSL, mARGOt autotuner 24] and the library for precision reduction and memoization provided by INRIA. Both parts of the system use the tools. On the server-side, the DSL is used to generate a part of the data access layer for the routing index stored in a HDF5 format [34]. It is also used to inject the integration code for the autotuner. mARGOt itselfis integrated in the Probabilistic Time-Dependent Routing (PTDR) algorithm in the routing pipeline 35$]$. It is used to estimate a number of Monte Carlo samples needed to obtain sufficiently precise estimation of the travel time. The autotuner is also used in a management process which facilitates communication with the actual routing workers to estimate number of workers of each type needed to satisfy current intensity of requests while conforming to a given SLA. Also the client side makes use of the mARGOt autotuner with the goal to optimize the requesting frequencies towards server-side services subject to constraints on navigation quality and data transfer limits. The ultimate goal is to alleviate server-side processing load to make system scalable and more robust across dynamic conditions.

b) Validation: In order to validate function of the serverside routing a traffic simulator has been developed. This

${ }^{11}$ http://heappe.eu 
software simulates cars driving around a given region along routes provided by the routing service 6 ]. A snapshot of such simulation in progress is shown in Figures. Global view on the traffic situation in this simulated traffic network is derived from the output of the simulator and fed back to the service by the generated probabilistic-speed profiles. This simulator will be used to prepare a set of deterministic scenarios with given traffic obstacles which will be used to validate the integrated tools. This part is the main experiment of the UC2, where the routing service will be validated along with the individual tools.

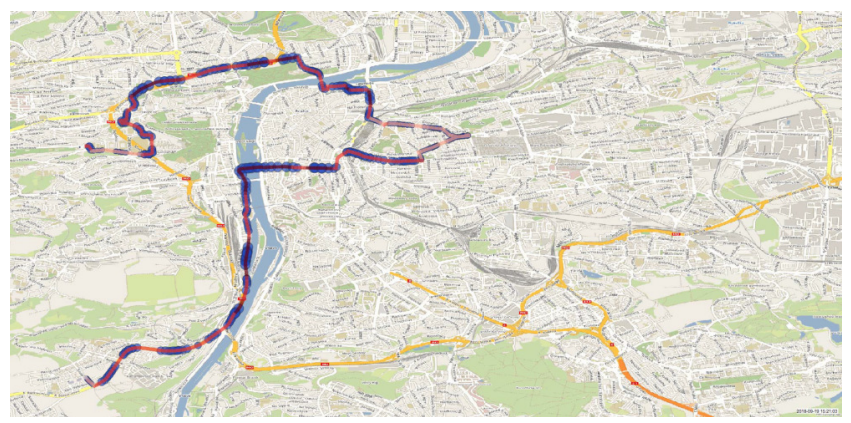

Fig. 5. Snapshot of the traffic simulation in progress, with both simulated floating car data (points) and state of the virtual traffic network (lines) made visible.

We expect that the routing service will optimize traffic for the specified scenarios such that all simulated cars will arrive at their destinations quicker when employing the serverside navigation. This will be tested by generating probabilistic speed profiles for each scenario with the given traffic event and comparing the results with simulation employing only a simple routing algorithm that is not aware of the traffic network state.

The next step is to validate the function of the autotuning tools. Operating points for the mARGOt autotuner used in the PTDR algorithm will be derived from the profiles, which in turn are derived from the traffic scenarios described above. The same scenario will be executed again with the autotuner We expect that it will not affect the SLA and the sum of Monte Carlo samples required for the entire simulation will be lower than in the case without any autotuning. Simulation logs will provide the timings for the individual requests passing through the stages of the routing pipeline. These data will be used to generate operating points for the autotuner used in the management process for workers autotuning. The same scenario will be executed again, this time with workers autotuning enabled. The expectation is that the SLA is still not violated, and that the number of running workers at any time during the simulation will be lower or similar than the number you get when the workers autotuning is enabled. Similarly the autotuner on the client-side, leveraging data transfer statistics of simulations and augmented with the definition of quality functions, will achieve comparable navigation quality using fewer requests to server-side services with respect to the baseline.
The Betweenness algorithm implementation utilizes tools developed by INRIA to reduce floating point precision and memoization. Small scale tests (single city) have been executed with promising results. The results are shown in Figure 6 . In the near future, we plan to execute large scale tests (entire region or country) on the Salomon cluster. We expect that tools provided by INRIA will improve the scalability of the algorithm while maintaining its sufficient precision.

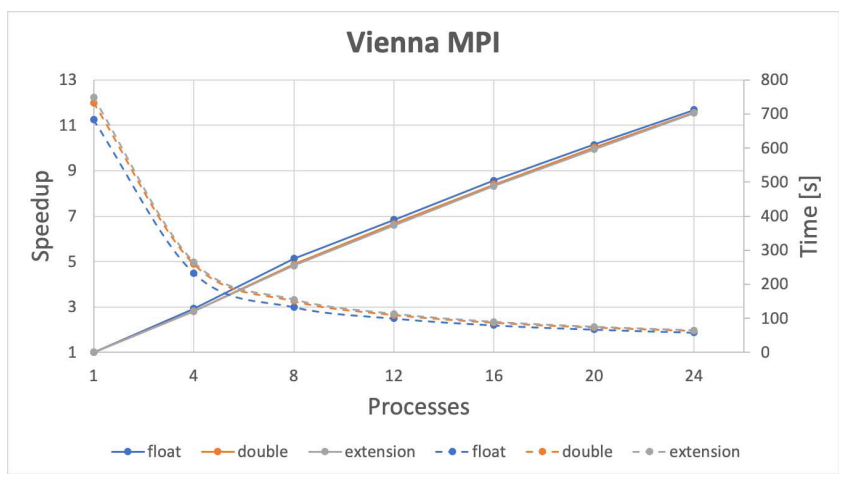

Fig. 6. Small scale test of the multi-node implementation of the betweenness algorithm executed on road network of Vienna with various floating point types.

\section{C ONCLUSIONS}

The ANTAREX project aims at addressing the increasing complexity of programming and tuning HPC applications through an approach based on decoupling the functional and extra-functional concerns. A DSL based on Aspect Oriented Programming principles serves as the vehicle to provide the programmer with a collection of tools to manage the applications' extra-functional properties, such as performance and energy. These properties are manager both at compiletime and at run-time, with an emphasis on supporting the autotuning of the application at run-time, based on the varying input data and system conditions. We presented the overall approach and the toolflow, and we then focused on the analyis of the performance issues that will be tackled by the scale-up experiment programmed by the two HPC centers, CINECA and IT4I. These experiments will be performed in the next months to fully demonstrate the capabilities of the ANTAREX software stack.

\section{ACKNOWLEDGEMENTS}

The ANTAREX project is supported by the EU H2020 FET-HPC program under grant 671623 . The IT4Innovations infrastructure is supported by the Large Infrastructures for Research, Experimental Development and Innovations project "IT4Innovations National Supercomputing Center LM2015070". The CINECA infrastructure is supported by the EU and the Italian Ministry for Education, University and Research (MIUR) under the PRACE project. 


\section{REFERENCES}

[1] C. Silvano, G. Agosta et al., "AutoTuning and Adaptivity appRoach for Energy efficient eXascale HPC systems: the ANTAREX Approach," in Design, Automation, and Test in EuropeDresden, Germany, Mar. 2016.

[2] C. Silvano, G. Agosta, S. Cherubin et al., "The ANTAREX approach to autotuning and adaptivity for energy efficient HPC systems," [2016 ACM Int'l Conf on Computing Frontiers, 2016, pp. 288-293.

[3] C. Silvano, A. Bartolini, A. Beccari, C. Manelfi, C. Cavazzoni, D. Gadioli, E. Rohou, G. Palermo, G. Agosta, J. Martinovi č et al., "The ANTAREX Tool Flow for Monitoring and Autotuning Energy Efficient HPC Systems (Invited paper)," in SAMOS 2017-Int'I Conf on Embedded Computer Systems: Architecture, Modeling and Simulation2017.

[4] C. Silvano, G. Agosta et al., "Antarex: A dsl-based approach to adaptively optimizing and enforcing extra-functional properties in high performance computing," inProceedings of the 2018 Euromicro Conference on Digital Systems Design2018.

[5] J. M. P. Cardoso, T. Carvalho, J. G. F. Coutinho, W. Luk, R. Nobre, P. Diniz, and Z. Petrov, "LARA: An Aspect-oriented Programming Language for Embedded Systems," inProc. 11th Annual Int'l Conf. on Aspect-oriented Software Development ACM, 2012, pp. 179-190.

[6] J. M. P. Cardoso, J. G. F. Coutinho, T. Carvalho, P. C. Diniz, Z. Petrov, W. Luk, and F. Gon ćalves, "Performance-driven instrumentation and mapping strategies using the LARA aspect-oriented programming ap proach," Software: Practice and Experience, Dec. 2014.

[7] J. M. Cardoso et al., "Lara: An aspect-oriented programming language for embedded systems," inProc. 11th Annual Int'l Conf. on Aspectoriented Software Development ACM, 2012, pp. 179-190.

[8] T. Kistler and M. Franz, "Continuous program optimization: A case study," ACM Transactions on Programming Languages and Systems (TOPLAS) , vol. 25, no. 4, pp. 500-548, 2003.

[9] S. Campanoni, M. Sykora, G. Agosta, and S. C. Reghizzi, "Dynamic look ahead compilation: a technique to hide jit compilation latencies in multicore environment," in International conference on compiler construction Springer, 2009, pp. 220-235.

[10] D. Binks, M. Jack, and W. Wilson, "Runtime compiled c++ for rapid ai development,"Game Al Pro: Collected Wisdom of Game Al Professionals, p. 201, 2013.

[11] S. Cherubin and G. Agosta, "libVersioningCompiler: An easy-to-use library for dynamic generation and invocation of multiple code versions," SoftwareX, vol. 7, pp. $95-100,2018$.

[12] R. Nobre, L. Reis, J. Bispo, T. Carvalho, J. M. P. Cardoso, S. Cherubin, and G. Agosta, "Aspect-driven mixed-precision tuning targeting gpus," in PARMA-DITAM '18, Jan 2018

[13] S. Cherubin, G. Agosta, I. Lasri, E. Rohou, and O. Sentieys, "Implications of Reduced-Precision Computations in HPC: Performance, Energy and Error," in Int'l Conf on Parallel Computing (ParCo), Sep 2017.

[14] D. Cattaneo, A. Di Bello, S. Cherubin, F. Terraneo, and G. Agosta, "Embedded Operating System Optimization through Floating to Fixed Point Compiler Transformation," in Proc of 2018 Euromicro Conference on Digital Systems Design, 2018.

[15] U. A. Acar, G. E. Blelloch, and R. Harper, "Selective memoization," in Proceedings of the 30th ACM SIGPLAN-SIGACT symposium on Principles of programming language,sser. POPL '03. New York, NY, USA: ACM, 2003, pp. 14-25.

[16] G. Agosta, M. Bessi, E. Capra, and C. Francalanci, "Dynamic memoization for energy efficiency in financial applications," inGreen Computing Conference and Workshops (IGCC), 2011 International IEEE, 2011, pp. 1-8.

[17] —-, "Automatic memoization for energy efficiency in financial applications," Sustainable Computing: Informatics and Systemsvol. 2, no. 2, pp. 105 - 115, 2012, iEEE International Green Computing Conference (IGCC 2011). [Online]. Available: http://www.sciencedirect. com/science/article/pii/S2210537912000066

[18] A. Suresh, E. Rohou, and A. Seznec, "Compile-Time Function Memoization," in 26th International Conference on Compiler Construction Austin, United States, Feb. 2017.

[19] A. Suresh et al., "Intercepting Functions for Memoization: A Case Study Using Transcendental Functions," ACM Trans. Archit. Code Optim. vol. 12, no. 2, p. 23, Jul. 2015.

[20] F. Beneventi, A. Bartolini, C. Cavazzoni, and L. Benini, "Continuous learning of hpc infrastructure models using big data analytics and in-memory processing tools," inDesign, Automation Test in Europe Conference Exhibition (DATE), 2017, March 2017, pp. 1038-1043.

[21] C. Silvano et al., "2parma: Parallel paradigms and run-time management techniques for many-core architectures," iD010 IEEE Computer Society Annual Symposium on VLS, July 2010, pp. 494-499.

[22] C. Silvano, W. Fornaciari, S. C. Reghizzi, G. Agosta, G. Palermo, V. Zaccaria, P. Bellasi, F. Castro, S. Corbetta, E. Speziale et al., "Parallel paradigms and run-time management techniques for many-core architectures: The 2parma approach," inndustrial Informatics (INDIN), 2011 9th IEEE International Conference on. IEEE, 2011, pp. 835-840.

[23] C. Silvano et al., "Multicube: Multi-objective design space exploration of multi-core architectures," in 2010 IEEE Computer Society Annual Symposium on VLSI July 2010, pp. 488-493.

[24] D. Gadioli, G. Palermo, and C. Silvano, "Application autotuning to support runtime adaptivity in multicore architectures," iEmbedded Computer Systems: Architectures, Modeling, and Simulation (SAMOS), 2015 Int'l Conf on. IEEE, 2015, pp. 173-180.

[25] D. Gadioli, E. Vitali, G. Palermo, and C. Silvano, "margot: a dynamic autotuning framework for self-aware approximate computingEEE Transactions on Computers 2018.

[26] A. Bartolini, R. Diversi, D. Cesarini, and F. Beneventi, "Self-aware thermal management for high performance computing processdE\&E Design \& Test, 2017.

[27] D. Cesarini, A. Bartolini, and L. Benini, "Prediction horizon vs. efficiency of optimal dynamic thermal control policies in hpc nodes," in 2017 IFIP/IEEE Int'I Conf on Very Large Scale Integration (VLSI-SoC) , Oct 2017, pp. 1-6.

[28] C. Cavazzoni, "EURORA: A European Architecture Toward Exascale," in Proc of the Future HPC Systems: The Challenges of PowerConstrained Performance New York, NY, USA: ACM, 2012, pp. 1:1-1:4

[29] A. Bartolini, M. Cacciari, C. Cavazzoni, G. Tecchiolli, and L. Benini, "Unveiling Eurora - Thermal and Power Characterization of the Most Energy-efficient Supercomputer in the World," irProc Conf. on Design, Automation \& Test in Europe 2014, pp. 277:1-277:6.

[30] "Mont-Blanc (FP7-ICT-2011-7 European project): European scalable and power efficient HPC platform based on lowpower embedded technology," 2011.

[31] "Mont-Blanc 2 (FP7-ICT-2013-10 European project), European scalable and power efficient HPC platform based on low-power embedded technology," 2013

[32] J. Hanzelka, M. B ěloch, J. Křenek, J. Martinovič, and K. Slaninová, "Betweenness propagation," infFIP International Conference on Computer Information Systems and Industrial ManagementSpringer, 2018, pp. 279-287.

[33] V. Svato ň, M. Podhoranyi, R. Vav řík, P. Veteška, D. Szturcová, D. Vojtek, J. Martinovič, and V. Vondrák, "Floreon+: A web-based platform for flood prediction, hydrologic modelling and dynamic data analysis," in Dynamics in Glscience, 2018, pp. 409-422.

[34] M. Golasowski, J. Bispo, J. Martinovi č, K. Slaninová, and J. M. Cardoso, "Expressing and applying c++ code transformations for the hdf5 api through a dsl," in IFIP Int'l Conf on Computer Information Systems and Industrial Management Springer, 2017, pp. 303-314.

[35] M. Golasowski, R. Tomis, J. Martinovi č, K. Slaninová, and L. Rapant, "Performance evaluation of probabilistic time-dependent travel time computation," inIFIP Int'I Conf on Computer Information Systems and Industrial Management Springer, 2016, pp. 377-388.

[36] V. Ptošek, J. Šev čík, J. Martinovi č, K. Slaninová, L. Rapant, and R. Cmar, "Real time traffic simulator for self-adaptive navigation system validation," in Proceedings of EMSS-HMS: Modeling \& Simulation in Logistics, Traffic \& Transportation, 2018. 